\section{Tuning sensory selection}

ERBB4
selectively
regulates the
strength of
the cortical
input to
the TRN

Faced with a constant barrage of sensory stimuli, the brain has to be selective: the most relevant stimuli must be processed, whereas irrelevant or distracting information should be ignored. Loss of such selective filtering is thought to occur in psychiatric disorders such as schizophrenia. Li and colleagues now provide insight into the circuitry that supports sensory selection and show that expression of the neuregulin 1 receptor ERBB4 is a key regulator of this process.

The thalamic reticular nucleus (TRN) contributes to sensory selection by modulating sensory information that passes through the thalamus. ERBB4 is expressed at high levels in the TRN; however, its function in this brain region is unknown. The authors generated

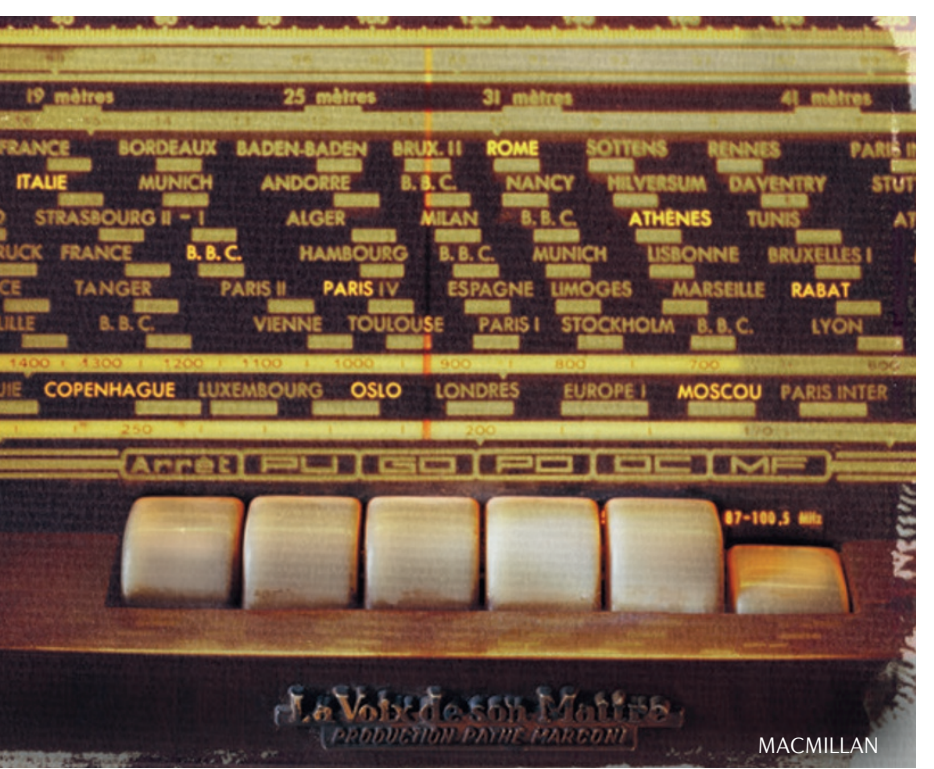

transgenic mice in which ERBB4 levels were specifically reduced or abolished in a large population of TRN neurons, and assessed the performance of these animals in two different behavioural tasks that assessed sensory selection.

In the first task, the animals learned to respond to a target sound and then to identify that sound among 'distractor' tones (a withinmodality task). In the second task, they initially learned to respond to sound cues and light cues, but were later required to respond only to the light, while ignoring the auditory stimuli - a task that involved switching attention across stimulus modalities. The mice lacking ERBB4 in TRN neurons demonstrated task, but reduced performance in the second, cross-modality task.

ERBB4 has roles in synapse formation and function in other brain regions and thus might influence synaptic function in the TRN. Indeed, the authors showed that excitatory post synaptic currents (EPSCs) recorded from TRN neurons lacking ERBB4 were larger than those in wild-type neurons, suggesting that ERBB4 weakens the strength of synapses onto these neurons. The TRN receives both cortical and thalamic input. To determine which of these synaptic inputs are affected by ERBB4 expression, the authors expressed channelrhodopsin-2 in neurons projecting to the TRN from either the thalamus or the cortex. Loss of ERRB4 affected only the TRN enhanced performance in the first and thalamic neuron responses to light-driven input from the cortex, suggesting that ERBB4 selectively regulates the strength of the cortical input to the TRN.

The authors further examined the functional effects of the changes in synaptic strength within the TRN by selectively weakening the synaptic input from the cortex to the TRN through overexpression of the carboxy-terminal tail of the AMPA receptor subunit GluA4 in TRN neurons. This reversed the effects of ERBB4 loss on performance in the two behavioural tasks, providing evidence that the effects of ERBB4 loss on sensory selectivity are mediated via changes in synaptic strength in the cortico-TRN pathway.

Cortical feedback to the thalamus may contribute to the suppression of responses to distracting stimuli; however, such feedback must be regulated to allow refocusing of attention where required (as in the second modality-switching task). These findings indicate that ERBB4 expression in the TRN has an important role in this tuning of cortical feedback to the thalamus, a function that may be relevant to the development of psychiatric disorders in which sensory selection is thought to be impaired.

Katherine Whalley

ORIGINAL RESEARCH PAPER Ahrens, S. et al. ErbB4 regulation of a thalamic reticular nucleus circuit for sensory selection. Nature Neurosci. http://dx.doi.org/10.1038/nn.3897 (2014) 